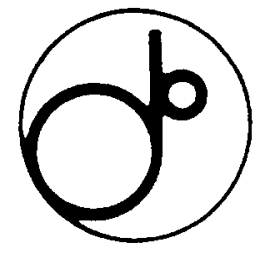

KEK Preprint 94-182

January 1995

A

\title{
1.5 MeV lon-Channel Guided X-band Free-Electron Laser Amplifier
}

K.Takayama, J. Kishiro, K.Ebihara, T.Ozaki, $\sec 9510$ S.Hiramatsu, and H.Katoh

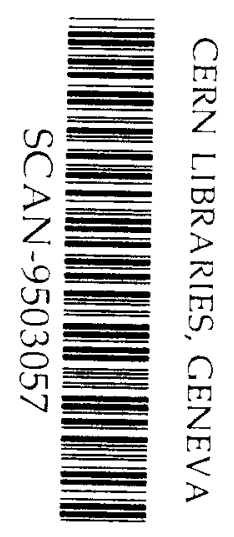

submitted to Applied Physics Letters and

presented at

The 4th Topical Meeting on Free Electron Laser and High Power

Radiation, Kyoto University, July 14-15, 1994

The 16th International Free Electron Laser Conference,

Stanford University, August 21-26, 1994

RF94: International Workshop on Pulsed RF Sources for Linear

Colliders, Montauk, Long Island, October 2-7, 1994

The 19th International Conference on Infrared and Millimeter

Waves, Sendai, October 17-20, 1994

The 4th Tamura Symposium on Accelerator Physics,

Austin, Texas, November 14-16, 1994 
National Laboratory for High Energy Physics, 1995

KEK Reports are available from:

Technical Information \& Library

National Laboratory for High Energy Physics

1-1 Oho, Tsukuba-shi

Ibaraki-ken, 305

JAPAN

Phone: $0298-64-1171$

Telex: $\quad 3652-534$

(0) $3652-534$ (International)

Fax: $\quad$ 0298-64-4604

Cable: $\quad$ KEK OHO

E-mail: LIBRARY@JPNKEKVX (Bitnet Address)

library@kekvax.kek.jp (Internet Address) 


\title{
1.5 MeV Ion-Channel Guided X-band Free- Electron Laser Amplifier
}

\author{
K.Takayama, J. Kishiro, K.Ebihara, T.Ozaki, S.Hiramatsu, H.Katoh \\ National Laboratory for High Energy Physics in Japan (KEK) \\ Oho 1-1, Tsukuba, Ibaraki, 305 Japan
}

Experiments on the Ion-Channel Guided X-band Free-Electron Laser Amplifier (IXFEL) generated peak microwave power exceeding $100 \mathrm{MW}$ at $9.4 \mathrm{GHz}$ with a gain of $21 \mathrm{~dB} / \mathrm{m}$. Saturation in the evolution curve has been achieved and a frequency spread of $0.9 \%$ was observed. The amplified microwave was separated from the driving beam line and extracted without break-down.

A microwave FEL has been regarded as a possible candidate of power sources for future linear colliders ${ }^{1}$ since the successful demonstration at $\mathrm{LLNL}^{2}$. While the Livermore experiment really triggered extensive theoretical studies on a multi-stage microwave $\mathrm{FEL}(\mu-\mathrm{FEL})$ in the so-called two-beam scheme at KEK and LBL/LLNL/MIT, other attractive applications of the $\mu-\mathrm{FEL}$ such as a planetary radar ${ }^{3}$, thunder-bolt control by atmosphere ionization ${ }^{4}$, or power transfer in space ${ }^{3}$ have become to be discussed on various occasions. Subsequent beam transport is crucial in the multi-stage regime. Ion channel guiding $(I C G)$ in the regime was proposed ${ }^{5}$, expecting Landau damping of beam break-up instability(BBU) and resistive-wall instability which are counter attacks for such long distance transport ${ }^{6}$. ICG technique was employed in the weakly relativistic $X$-band $F E L(800 \mathrm{keV})^{7}$ which was our preliminary set-up. Other crucial problems are seed power injection into the beam line and amplified-power extraction from it with sufficient efficiency. In fact a large magnitude of the seed power has been theoretically proved to mitigate outputphase's sensitivity to injection errors in driving beam current or accelerating voltage ${ }^{8}$. Recently we have developed over-sized input and output couplers 
with efficiency of nearly $90 \%$ in TE01 mode which can minimize reflection and mode conversion. The present IXFEL integrates the ICG, conventional planar wiggler and RF handling system with efficient $\mu$-couplers.

As schematically shown in Fig.1, an input signal from a pulsed magnetron(EEV M5188) is fed into the over-sized rectangular waveguide (RW, WRJ-2, $5.5 \mathrm{~cm} \times 11 \mathrm{~cm}$ ) with a miter-bend with a small hole of $20 \mathrm{~mm} \phi$ in diameter. The amplified microwave is coupled out from the driving-beam line with the other miter-bend. A fraction of $\mu$-waves eventually emitted into the anechoic room with a horn-antenna is received with a basic-size open-RW placed in the well-aligned forward direction. The radiation signal is attenuated down to the milliwatt level and monitored by a crystal diode. The used attenuators were periodically checked for reproducibility. Modeconversion in over-sized RF components was measured by the phase interference method ${ }^{9}$ where a far-field pattern of the transmitted reference signal from a Gunn-oscillator is monitored as a function of the straight-RW length being varied over a wavelength of beatwave between the dominant TE01 mode and possible higher mode such as TE03. Both of the input and output coupler demonstrated a transmission efficiency of $85 \%$ in TE01 mode, giving a net seed-power of $77 \mathrm{~kW}$, which is consistent with the MAFIA simulation $^{10}$. A pressure of Diethylaniline(DEA) gas filling the beam-line is monitored by four Shultz and BA gauges calibrated to a Baratron capacitance manometer. An KrF excimer laser(18nsec pulse length, $\lambda=248 \mathrm{~nm}$, $140 \mathrm{~mJ} / 20 \mathrm{~mm} \phi$ with shot-to-shot jitters of $5 \mathrm{~mJ}$.) is used to ionize the DEA by the two-photon resonant process. An ion-density of $2 \times 10^{10} \mathrm{~cm}^{-3}$ in typical operation was extrapolated from two independent informations ${ }^{9}$ : a magnitude of ion-channel relaxation in the relativistic Langmuir-Child limited beam current which straightforwardly reflects the ion-density and a critical iondensity at which beam's envelope hits the waveguide's wall $2.5 \mathrm{~m}$ downstream from the emittance selector. Details of the induction gun energized with two 
magnetic switches were given elsewhere ${ }^{11}$. A good emittance region of $650 \mathrm{~A}$ was diged out of an arriving 1.3kA e-beam with this emittance selector which is simply a combination of ion channel and narrow $1 \mathrm{~m}$-long aluminum pipe of $20 \mathrm{~mm} \phi$ in diameter. The emittance selector has an acceptance of .06-.04 cmrad $^{12}$ for a fixed gas pressure and laser intensity. In the maximum amplification regime mentioned later, a beam current of $450 \mathrm{~A}$ was monitored at the wiggler end. Each unit of the air-core planar wiggler magnet $\left(\lambda_{w}=16 \mathrm{~cm}\right.$ and 15 periods) is independently energized with a pulse power-supply and the wiggler's field-uniformity ( $\triangle \mathrm{BW} / \mathrm{BW}=1.5 \%$ at the RW's horizontal edge $)^{13}$ is provided with thick copper bars inserted in both sides of the wiggler gap, eddy currents on which protects flux-leakage in the horizontal direction.

Typical examples of the amplified signal for two different wiggler fields $(1.21 \mathrm{kG}$ and $.93 \mathrm{kG})$ are shown in Fig.2. Resonant structure in the pulse duration clearly indicates the existence of resonant beam energy. Two resonant portions in time merges at the central position in pulse beyond $B w=1.2 k G$ which corresponds to the peak energy position.

Frequency spectrum(FS) of the amplified signal was measured by a low- $Q$ transmission-type frequency counter, the filling time $(2 Q / \omega)$ of which is about 4 nsec to be much shorter than the pulse duration of 10-15nsec. Fig.3 represents the FS of the seed and amplified signals at $B w=1.21 \mathrm{kG}$. The frequency spread $\mathrm{s}$ of $\Delta \mathrm{f} / \mathrm{f}=0.6 \%$ and $0.9 \%$ in $\mathrm{FWHM}$ were found, respectively. The former was set by a finite $Q$-value of the frequency counter, that is, 120 , because that of the seed pulse obtained by a spectral analyzer is less than $0.1 \%$ in FWHM. Another notable result of frequency measurements was a shift in the FS for the resonant portion which corresponds to FEL interaction of lower-energy beams in a lower wiggler field just mentioned above; in a case of $\mathrm{Bw}=.93 \mathrm{kG}$ the $\mathrm{FS}$ is downshifted by $\sim 60 \mathrm{MHz}$, as seen in Fig.3. Reasonable explanation for frequency-broadning and the shift has not been found yet. 
Dependence of the gain on wiggler field was measured at the position just before saturation which will be mentioned later. In Fig.4, a magnitude of the amplified power is shown as a function of the wiggler field. Indicated data here were taken at the same pulse timing that gives the peak power for $B w=1.2-1.25 \mathrm{kG}$. A fractional magnetic field "bandwidth", $\triangle B w / B w$, in FWHM is about $8.1 \%$. Beam transmission efficiency gradually degraded beyond $\mathrm{Bw}=1.2 \mathrm{kGauss}$. Therefore, the detuning curve should be somewhat different from an ideal case without beam loss. This speculation seems to be consistent with some estimation from the theoretical detuning curves shown in the same figure where a lower beam current certainly gives much small gain in the region of higher wiggler field. Assuming possible transmission beam currents $(I=450,200 A)$ and an energy-spread of $1.5 \%$, these detuning curves are derived from a cubic dispersion relation taking account of space-charge effects, geometrical coupling with TE01 mode, vertical betatron motion and quadratic wiggler field variation in the vertical direction which depend on a spatial size in the RW effectively occupied by beams ${ }^{9}$.

Evolution curve was obtained by simply turning on the wiggler unit in order. Evolutions for RW-length covering a beatwave length between TE01 and TE03 are depicted in Fig.5. The evolution curve adjusted by the phase interference method which places roughly in the middle of scattered points indicates the exponential gain of $21 \mathrm{~dB} / \mathrm{m}$. Apparently, TE01 mode dominates the received power. A small fraction of the received power originates from TE03 mode converted in the output coupler including the RF-window. The experimentally obtained size of the gain is in good agreement with theoretical estimation mentioned in the previous paragraph. For power measurements of possibly evolving TE21 mode, the receiver horn was positioned at $\theta=20^{\circ}$ less than the ideal location of $\theta=\lambda / b \approx 33^{\circ}$ because of a finite-size of the anechoic room. The signal level was not discriminated from that of the dominant mode. 
Theoretical calculation based on the cubic dispersion relation ${ }^{9}$ also tells us that the gain for the pure TE21 mode is quite small.

A systematic study on effects of the ion channel on FEL amplification was not easy. Since there should be no change in the refraction index in the underdense regime $\left(n_{i}<n b\right)$ of our interest, the FEL condition depends on the iondensity only through a change in betatron-frequency. We observed that the gain was largely reduced, accompanied with significant beam-loss, in lower and higher pressure regions than the nominally operating pressure. This indicates that beam transport through the wiggler region takes a trivial but practical role in FEL amplification.

By integrating ion-channel guiding, ion-channel guided emittance selection, the eddy-current assisted wiggler, and efficient miter RF-bends, the reasonable $\mu-F E L$ amplification was achieved with effective saturation in a reliable manner. The IXFEL has demonstrated the viability of the ICG for future interesting applications beyond two proceeding ICG experiments ${ }^{14}$.

The authors wish to thank Associate Director, Prof. Y.Kimura, for his continuous support to our R\&D works. K.Saitoh and S. Hashimoto contributed to early works of magnetic field measurement and $\mu$-wave calibration, respectively. 


\section{References}

1 A.M.Sessler, Laser Acceleration of Particles (Los Alamos, 1982), Proc. of the Conf. on Laser Acceleration of Particles, AIP Proceedings 91, edited by P.J.Channel(AIP, New York, 1982), p.163.

2 T.J.Orzechowski et al., Phys. Rev. Lett. 57, 2172 (1986).

3 K.Takayama, S. Hiramatsu, and M.Shiho, J. of British Interplanetary Soc. 44, 573 (1991).

4 M.Shiho et al., Digest of 19th Int. Conf. on Infrared and Millimeter Waves in Sendai (1994), p.254.

5 K.Takayama and S. Hiramatsu, Phys. Rev. A 37, 173 (1988).

6 K.Takayama, Phys. Rev. A 39, 184 (1989), D.H.Whittum, A.M.Sessler, and V.K.Neil, Phys. Rev. A 43, 294 (1991).

7 T.Ozaki et al., Nucl. Inst. and Meth. A318, 101 (1992), T.Monaka, Ph.D Thesis (1992).

8 K.Takayama, Part. Accel. 39, 65 (1992).

9 K.Takayama et al., to be published.

10 K.Takayama et al., Nucl. Inst. and Meth. A341, 109 (1994).

11 J.Kishiro et al., Proc. of 1993 Accelerator Conf. in Washington (1993), p673.

12 An acceptance of the emittance selector is given by the formula,

$\sqrt[r]{\frac{2 I}{\gamma l_{A}}\left(f-\frac{1}{\gamma^{2}}\right)}$ where $r$ is the pipe radius, $f$ is the neuralization factor, I and $\phi_{A}$ are the beam current and Alfven current, respectively.

13 K.Takayama et al., KEK preprint 89-152 (1989), unpublished.

14 W.E.Martin et al., Phys. Rev. Lett. 54, 685 (1985), G.J.Caporaso et al., Phys. Rev. Lett. 57, 1591 (1986), S.L.Shope et al., Phys. Rev. Lett. 58, 551 (1987). 


\section{Figure Captions}

Fig. 1 Schematic view of the IXFEL.

Fig.2 Amplified signals at $\mathrm{Bw}=0.93 \mathrm{kG}$ (solid) and $1.21 \mathrm{kG}$ (dash), Beam voltage profile in time(lower trace).

Fig.3 Frequency spectrum of the amplified signal at $B w=1.21 \mathrm{kG}$ (solid) and at $\mathrm{Bw}=0.93 \mathrm{kG}$ (long dash), magnetron signal(short dash), by a spectral analyzer(shortest dash).

Fig.4 Experimental detuning curve and theoretical calculations for $\mathrm{I}=450 \mathrm{~A}$ (long dash) and 200A(short dash).

Fig.5 Evolution for different RW-lengths and the adjusted TE01 evolution curve. 


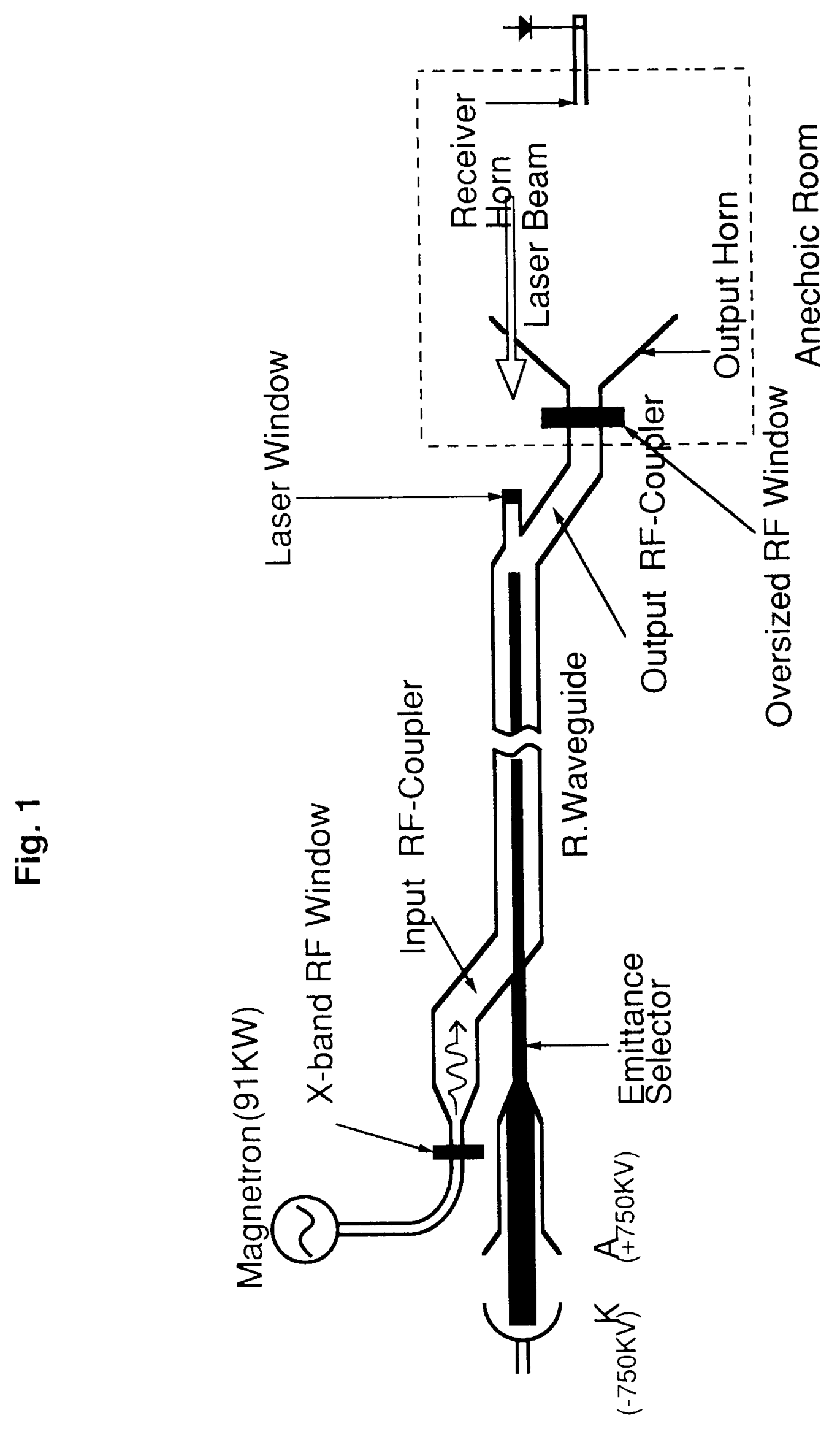




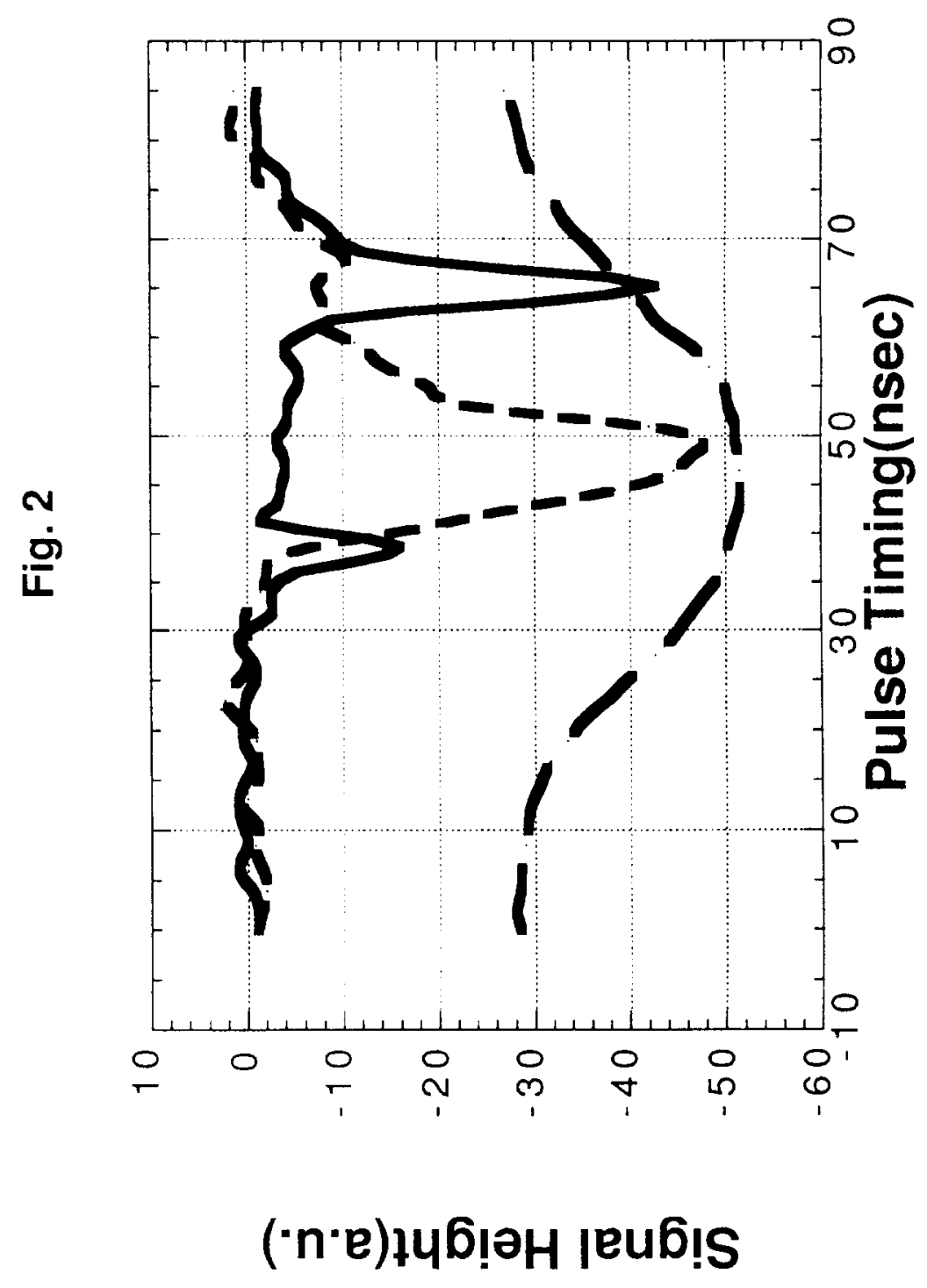




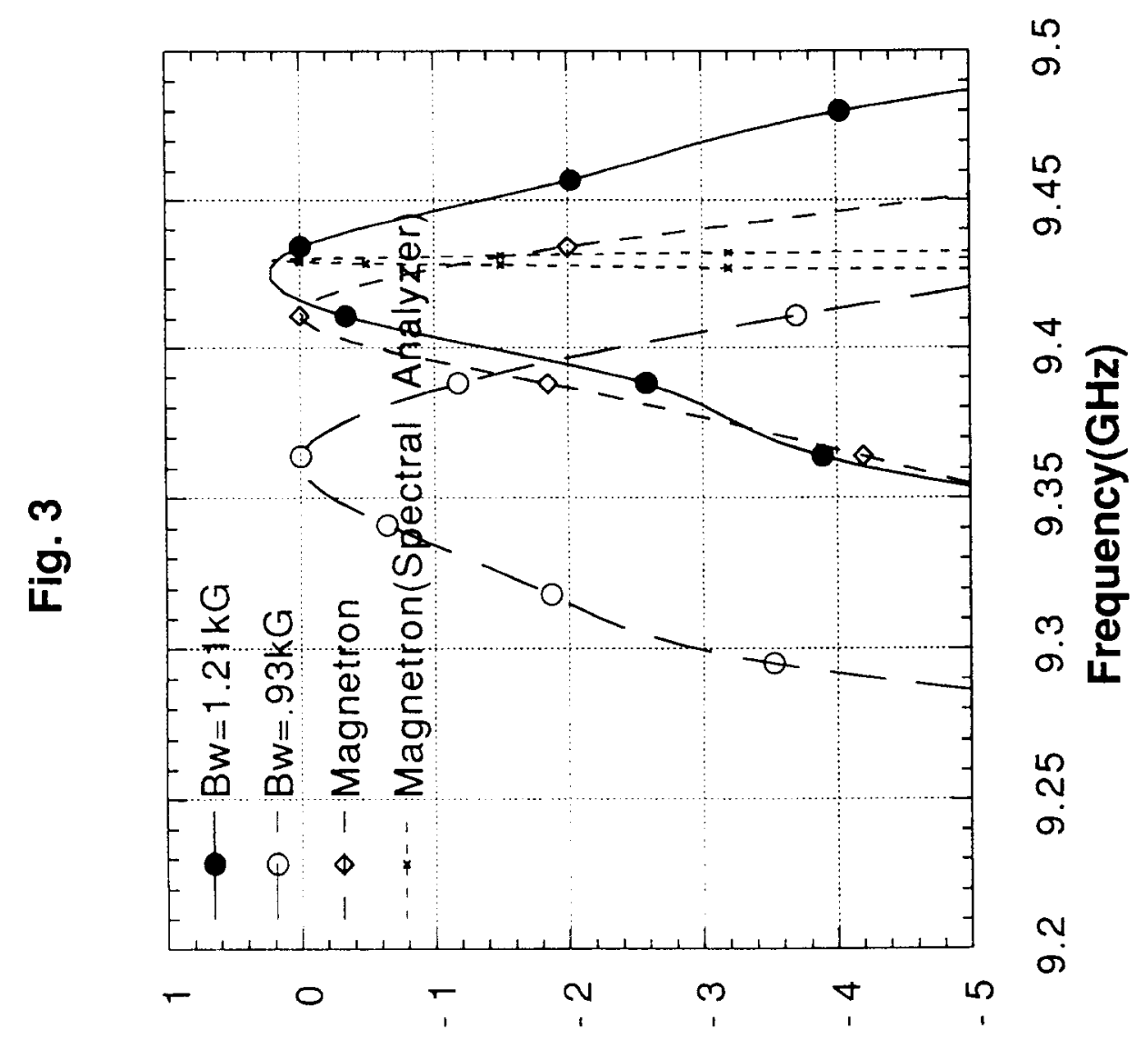

(gp)גәмод 


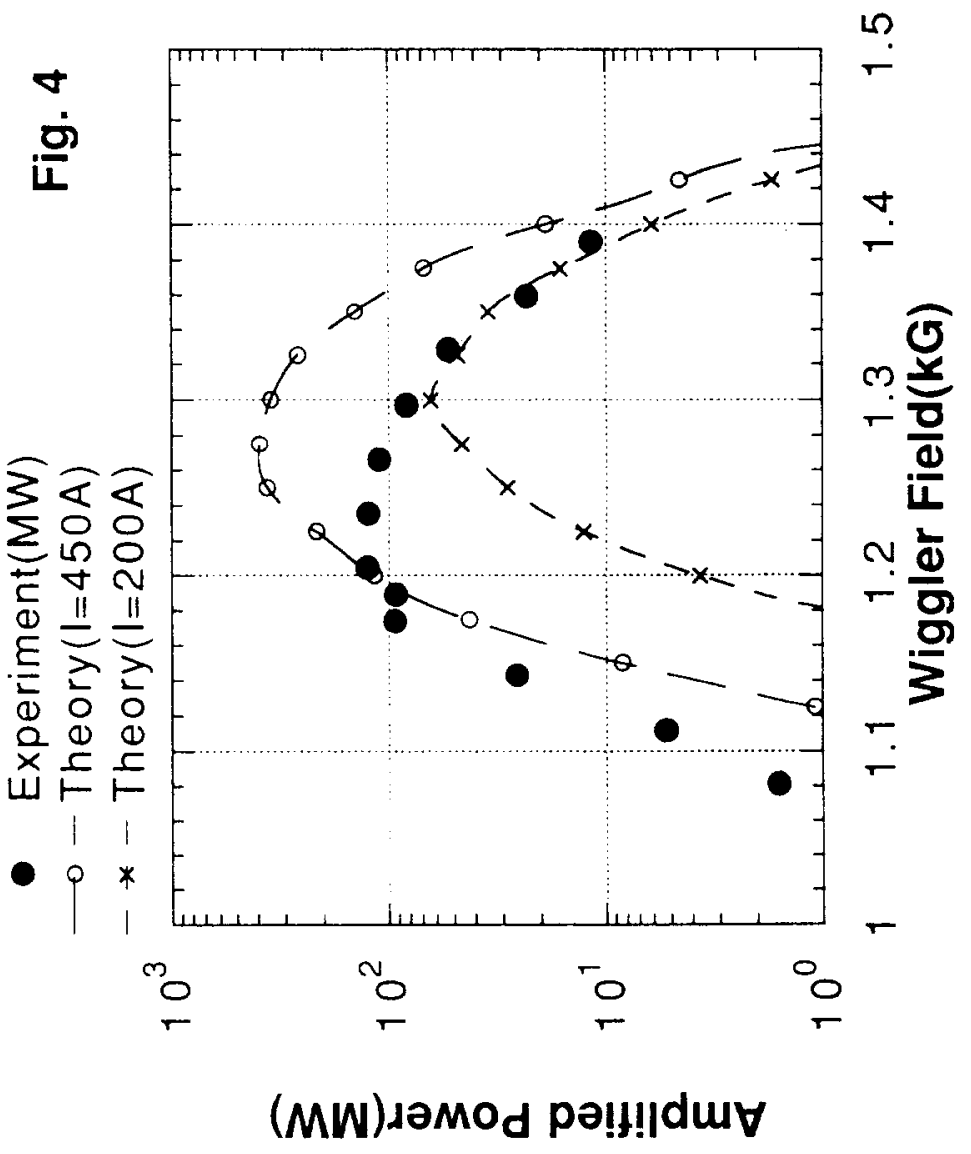




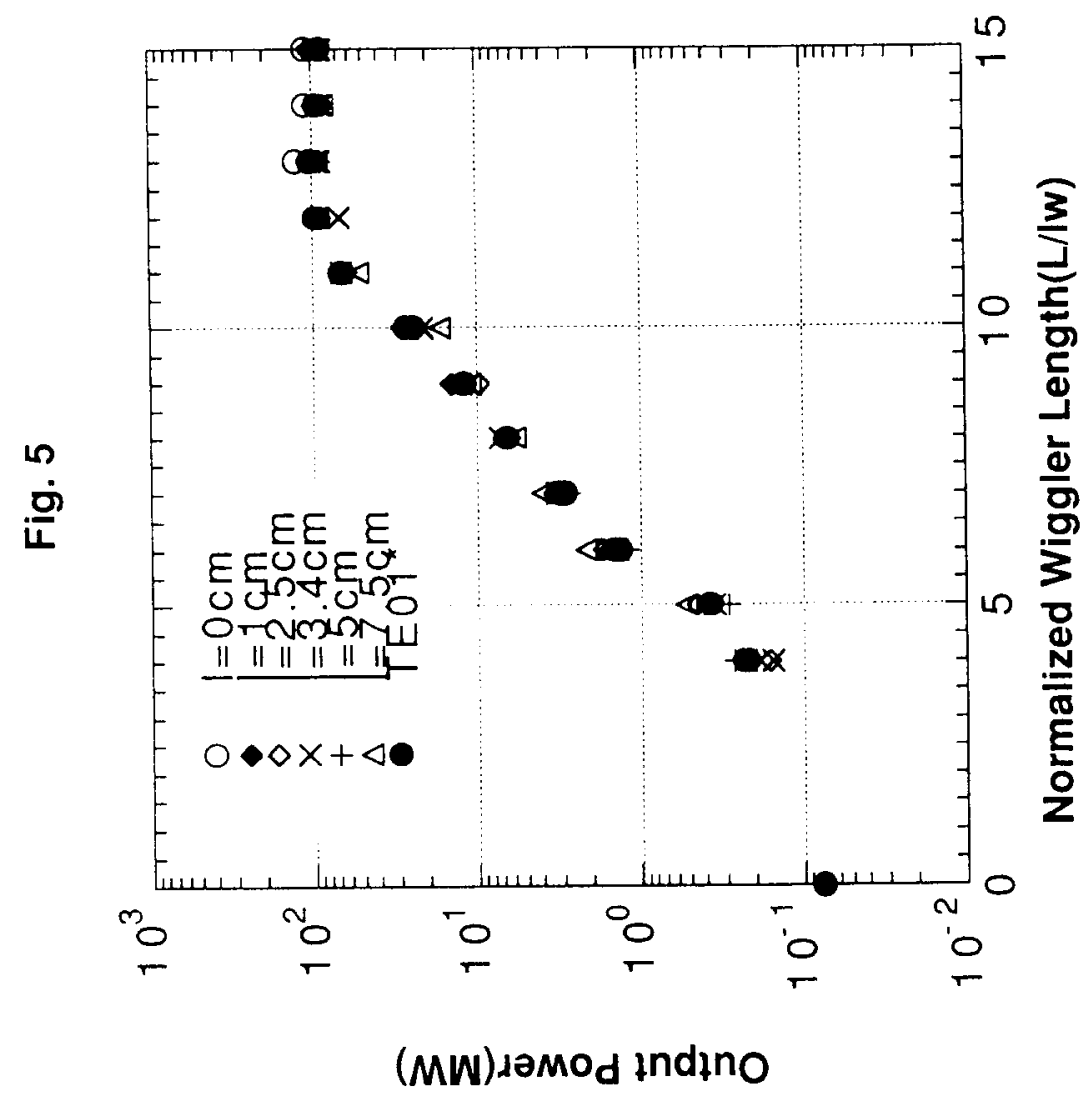

$-12-$ 